\title{
Supersymmetric quantum mechanical generalized MIC-Kepler system
}

\author{
Pulak Ranjan Giri* \\ Saha Institute of Nuclear Physics, 1/AF Bidhan-Nagar, Calcutta 700064, India
}

September 26, 2018

\begin{abstract}
We construct supersymmetric(SUSY) generalized MIC-Kepler system and show that the systems with half integral Dirac quantization condition $\mu= \pm \frac{1}{2}, \pm \frac{3}{2}, \pm \frac{5}{2}, \ldots$. belong to a SUSY family (hierarchy of Hamiltonian) with same spectrum between the respective partner Hamiltonians except for the ground state. Similarly, the systems with integral Dirac quantization condition $\mu= \pm 1, \pm 2, \pm 3, \ldots \ldots$ belong to another family. We show that, it is necessary to introduce additional potential to MIC-Kepler system in order to unify the two families into one. We also reproduce the results of the (super-symmetric) Hydrogenic problem in our study.

Keywords: SUSY quantum mechanics, generalized MIC-Kepler system, bound states

PACS numbers: 11.30.Pb; 03.65.-w; 11.30.-j
\end{abstract}

\section{Introduction}

Supersymmetric (SUSY) quantum mechanics is getting more attention and is being studied extensively nowadays. However, the concept of SUSY came in High Energy Physics for the first time in order to obtain all the fundamental interactions of nature [1, 2, 3, 4, 5, under a sort of unified theory. SUSY is a highly nontrivial symmetry for fermions and bosons. Because, fermions and bosons are particles with different principle and statistics, for example, fermion obeys Pauli exclusion principle but boson does not. SUSY has some predictions in High Energy Physics. For example, every fundamental particle (i.e., quarks, leptons, gluon, photon, etc.) in nature must have its SUSY partner, whose spins differ by halfintegral but mass are equal when the symmetry is preserved. But so far there is no experimental evidence that SUSY is preserved in nature. Then people started to think about SUSY breaking. Inspired by this fact, Witten [6] started to study SUSY breaking in non-relativistic quantum mechanics as a toy model for SUSY breaking in quantum field theory. SUSY has now application in atomic, nuclear, condensed matter and statistical physics 7 also.

SUSY quantum mechanics has now become a separate field of study and has received lot of interest for its beautiful mathematical insight as well as for various aspect of non-relativistic quantum mechanics. Non-relativistic coulomb problem is a standard problem in SUSY quantum mechanics, for example. In Ref. [8] it has been shown that the distinct spectrum of certain atoms and ions have quantum mechanical SUSY. For example, the $s$ levels of the lithium atom can be interpreted as the SUSY partner of the hydrogen atom $s$ levels in the absence of electron-electron interactions and provided the valence electron of lithium is far enough removed from the core electrons cloud. It is also discussed that the SUSY is broken if electron-electron interaction is present for the obvious reason that the level with fixed principal

*e-mail :pulakranjan.giri@saha.ac.in 
quantum number $n$ and different orbital angular momentum quantum number $l$ splits up. For other discussion on coulomb problem see Ref. [9, 10, 11, 12]. Later it is however shown that the relativistic Coulomb problem also have exact SUSY [13].

So far known knowledge of SUSY on Coulomb problem naturally raises the question whether it can be extended to the case of two dyon system (MIC-Kepler). Because MIC-Kepler system [14, 15, is very similar to the non-relativistic coulomb problem, where two dyons with electric and magnetic charges $e_{1}, g_{1}$ and $e_{2}, g_{2}$ respectively are present and the radial equations of both the system are exactly equal except the parameter orbital angular momentum. Not only that, MIC-Kepler system restores all the symmetry of non-relativistic Coulomb system like $O(4)$ for bound state and $O(1,3)$ for continuum state if a potential of the form $\sim \frac{1}{r^{2}}$ is added to the Hamiltonian by hand. The system has been further generalized [16, 17, and it is called generalized MIC-Kepler system for obvious reason. In this present work we will discuss about this generalized MIC-Kepler system [16, 17] in the framework of SUSY quantum mechanics.

The paper is organized as follows: In Sec. 2, we recapitulate the basic formalism of SUSY quantum mechanics. In Sec. 3 we will discuss about the generalized MIC-Kepler system. Because in order to discuss the SUSY of the model it is necessary to know about the details of the model. Then in Sec. 4. the SUSY extension of the generalized MIC-Kepler system will be discussed and some observations will be made. Finally we conclude in Sec. 5 .

\section{General review of the formalism of SUSY quantum mechanics}

In this section we recapitulate the general feature of SUSY quantum mechanics. For detail review see [7, 18, 19, 20, 21] and the references therein. The SUSY quantum mechanical Hamiltonian can be written as

$$
H_{\text {susy }}(x)=H_{+}(x) \oplus H_{-}(x) .
$$

In $\hbar=m=1$ unit, the two partner Hamiltonians $H_{+}(x)$ and $H_{-}(x)$ take the form

$$
H_{ \pm}(x)=-\frac{1}{2} \frac{d^{2}}{d x^{2}}+V_{ \pm}(x)
$$

where the SUSY partner potentials $V_{+}(x)$ and $V_{-}(x)$ can be written in terms of superpotential $W(x)$ as

$$
V_{ \pm}(x)=W^{2}(x) \mp \frac{1}{\sqrt{2}} W^{\prime}(x),
$$

It can be easily checked from Eq. (2.2) and Eq. (2.3) that the Hamiltonian $H_{+}(x)$ and $H_{-}(x)$ can be factorized in the form

$$
H_{+}(x)=A(x)^{\dagger} A(x), \quad H_{-}(x)=A(x) A(x)^{\dagger},
$$

if we consider the first order differential operator $A(x)$ and $A(x)^{\dagger}$ of the form

$$
A(x)=\frac{1}{\sqrt{2}} \frac{d}{d x}+W(x), A^{\dagger}(x)=-\frac{1}{\sqrt{2}} \frac{d}{d x}+W(x) .
$$

We can define two supercharges $Q(x)$ and $Q(x)^{\dagger}$ of the form

$$
Q(x)=A(x) \sigma_{-}, \quad Q^{\dagger}(x)=A^{\dagger}(x) \sigma_{+},
$$

where $\sigma_{+}$and $\sigma_{-}$can be written in terms of Pauli matrices as

$$
\sigma_{ \pm}=\frac{1}{2}\left(\sigma_{x} \pm i \sigma_{y}\right)
$$


The Hamiltonian $H_{\text {susy }}(x)$ together with the two supercharge $Q(x)$ and $Q^{\dagger}(x)$ form the closed superalgebra $\operatorname{sl}(1,1)$ :

$$
\begin{aligned}
{\left[H_{\text {susy }}(x), Q(x)\right] } & =\left[H_{\text {susy }}(x), Q^{\dagger}(x)\right]=0,\left\{Q(x), Q^{\dagger}(x)\right\}=H_{\text {susy }}(x), \\
\{Q(x), Q(x)\} & =\left\{Q^{\dagger}(x), Q^{\dagger}(x)\right\}=0 .
\end{aligned}
$$

The SUSY partner Hamiltonians $H_{+}(x)$ and $H_{-}(x)$, its eigenvalues $E_{n}^{(+,-)}$and the eigenfunctions are related among themselves if it is shown that SUSY is preserved for a quantum mechanical system. The time independent Schrödinger eigenvalue equation for $H_{+}(x)$ takes the form

$$
H_{+}(x) \psi_{n}^{+}(x)=A^{\dagger}(x) A(x) \psi_{n}^{+}(x)=E_{n}^{+} \psi_{n}^{+}(x) .
$$

The Hamiltonian $H_{-}(x)$ has also the same eigenvalue $E_{n}^{+}$but the eigenfunction is different. It is easily found by multiplying from left on both sides of equation (2.9) by the operator $A$

$$
H_{-}(x)\left(A(x) \psi_{n}^{+}(x)\right)=A(x) A^{\dagger}(x) A(x) \psi_{n}^{+}(x)=E_{n}^{+}\left(A(x) \psi_{n}^{+}(x)\right) .
$$

It is also possible to write the time independent Schrödinger eigenvalue equation for $H_{-}(x)$ with eigenvalue $E_{n}^{-}$and eigenfunction $\psi_{n}^{-}$and from there one can show that $H_{+}(x)$ has also the same eigenvalue $E_{n}^{-}$but with eigenfunction $A^{\dagger}(x) \psi_{n}^{-}(x)$.

$$
\begin{gathered}
H_{-}(x) \psi_{n}^{-}(x)=A(x) A^{\dagger}(x) \psi_{n}^{-}(x)=E_{n}^{-} \psi_{n}^{-}(x) . \\
H_{+}(x)\left(A^{\dagger}(x) \psi_{n}^{-}(x)\right)=A^{\dagger}(x) A(x) A^{\dagger}(x) \psi_{n}^{-}(x)=E_{n}^{-}\left(A^{\dagger}(x) \psi_{n}^{-}(x)\right) .
\end{gathered}
$$

It is clear that the same eigenvalue is shared by the two partner Hamiltonians $H_{+}(x)$ and $H_{-}(x)$ but with different eigenfunctions. The exception occurs when some eigenfunction is annihilated by $A(x)$. It is obvious that the eigenfunction annihilated by the operator $A(x)$ is a ground state, but ground state may not be annihilated by $A(x)$. The above equation (2.10) shows that $\psi_{0}^{+}(x)$ is the ground state, which may or may not be annihilated by $A(x)$, i.e., $A(x) \psi_{0}^{+}(x)=0$, or $\neq 0$.

All eigenstates of the two partner Hamiltonians are paired when the ground state $\psi_{0}^{+}(x)$ is not annihilated by $A(x)$. The exact relation between the eigen-values and eigen-functions are easily found to be

$$
E_{n}^{-}=E_{n}^{+}>0, \quad \psi_{n}^{-}(x)=\left[E_{n}^{+}\right]^{-1 / 2} A(x) \psi_{n}^{+}(x), \quad \psi_{n}^{+}(x)=\left[E_{n}^{-}\right]^{-1 / 2} A^{\dagger}(x) \psi_{n}^{-}(x) .
$$

But this does not happen when the ground state $\psi_{0}^{+}(x)$ is annihilated by $A(x)$. In this case the ground state remains unpaired, while all other states of the two partner Hamiltonians are paired. This time the exact relation between the eigen-values and eigen-functions are found to be

$$
E_{n}^{-}=E_{n+1}^{+}, \quad E_{0}^{+}=0, \quad \psi_{n}^{-}(x)=\left[E_{n+1}^{+}\right]^{-1 / 2} A(x) \psi_{n+1}^{+}(x), \quad \psi_{n+1}^{+}(x)=\left[E_{n}^{-}\right]^{-1 / 2} A^{\dagger}(x) \psi_{n}^{-}(x) .
$$

Note that when the ground state $\psi_{0}^{+}(x)$ is annihilated by $A(x)$, the expression for the ground state can be found if the super-potential $W(x)$ is known. On the other hand if the ground state is known then the expression for the super-potential can be found from $A(x) \psi_{0}^{+}(x)=0$. Depending on which one is given the ground state and the super-potential are given respectively as follows:

$$
\begin{aligned}
& \psi_{0}^{+}(x) \sim \exp \left[-\sqrt{2} \int^{x} W(y) d y\right] . \\
& W(x)=-2^{-\frac{1}{2}} \psi_{0}^{\prime+}(x)\left(\psi_{0}^{+}(x)\right)^{-1} .
\end{aligned}
$$

One can show that the above mentioned procedure can in fact be repeatedly used in order to generate a hierarchy of Hamiltonians [18, 19, 20, 21. For example starting with the original Hamiltonian $H_{1}$, which has $p(\geq 1)$ bound states with eigenvalues $E_{n}^{(1)}$ and eigenfunctions $\psi_{n}^{(1)}$ with $0 \leq n \leq(p-1)$, a hierarchy of $(p-1)$ Hamiltonians $H_{2}, H_{3}, \ldots H_{p}$ can be constructed so that the m'th member of the hierarchy of Hamiltonians $\left(H_{m}\right)$ have the same eigenvalue as $H_{1}$ except that the first $(m-1)$ eigenvalues of $H_{1}$ are missing in $\left(H_{m}\right)$. 


\section{Generalized MIC-Kepler system}

In introduction we mentioned that generalized MIC-Kepler system [16, 17] is basically dynamics of two dyon system. The Hamiltonian of the system is given by (in system of units $\hbar=m=c=1$ )

$$
H=\frac{1}{2}(-i \nabla-\mu \mathbf{A})^{2}+\frac{\mu^{2}}{2 r^{2}}-\frac{1}{r}+\frac{c_{1}}{r^{2}(1+\cos \theta)}+\frac{c_{2}}{r^{2}(1-\cos \theta)},
$$

Here $\mathbf{A}$ is the magnetic vector potential of the Dirac monopole, given by

$$
\mathbf{A}=-\frac{\sin \theta}{r(1-\cos \theta)} \hat{\phi}
$$

such that $\operatorname{curl} \mathbf{A}=\frac{\mathbf{r}}{r^{3}} . c_{1}, c_{2}$ are nonnegative constants and $\mu$, the Dirac quantization condition takes the values $0, \pm \frac{1}{2}, \pm 1, \cdots$.

This system has been generalized from MIC-Kepler [14, 15] system by adding two axially symmetric potentials with coefficients $c_{1}$ and $c_{2}$. It has been solved in spherical and parabolic coordinates [16, 17] and even generic solution [22] has been considered by making a one parameter family of self-adjoint extensions. Here we describe the solution in spherical polar coordinates in order to get an idea of the generalized MIC-kepler system, which we will need in the next section for super-symmetric extension of the system.

The Schrödinger eigenvalue equation is

$$
H \Psi=E \Psi,
$$

where the Hamiltonian $H$ is given in Eq. (3.1). We can separate Eq. (3.3) in spherical polar coordinates $(r, \theta, \varphi)$ using the wave-function $\Psi$ of the form

$$
\psi(r, \theta, \varphi)=R(r) Z(\theta, \varphi)
$$

Substituting Eq. (3.4) in the Schrödinger eigenvalue equation Eq. (3.3) one can get angular and radial part of the differential equation completely separated. The angular differential equation takes the form

$$
\frac{1}{\sin \theta} \frac{\partial}{\partial \theta}\left(\sin \theta \frac{\partial Z}{\partial \theta}\right)+\frac{1}{4 \cos ^{2} \frac{\theta}{2}}\left(\frac{\partial^{2}}{\partial \varphi^{2}}-4 c_{1}\right) Z+\frac{1}{4 \sin ^{2} \frac{\theta}{2}}\left[\left(\frac{\partial}{\partial \varphi}+2 i \mu\right)^{2}-4 c_{2}\right] Z=-\mathcal{A} Z,
$$

where the separation constant $\mathcal{A}$ is quantized as

$$
\mathcal{A}=\left(j+\frac{\delta_{1}+\delta_{2}}{2}\right)\left(j+\frac{\delta_{1}+\delta_{2}}{2}+1\right) .
$$

The solution of Eq.(3.5) can be found apart from normalization as

$$
Z_{j m}^{(\mu)}\left(\theta, \varphi ; \delta_{1}, \delta_{2}\right)=\left(\cos \frac{\theta}{2}\right)^{m_{1}}\left(\sin \frac{\theta}{2}\right)^{m_{2}} P_{j-m_{+}}^{\left(m_{2}, m_{1}\right)}(\cos \theta) e^{i(m-\mu) \varphi},
$$

where $m_{1}=|m-\mu|+\delta_{1}=\sqrt{(m-\mu)^{2}+4 c_{1}}, m_{2}=|m+\mu|+\delta_{2}=\sqrt{(m+\mu)^{2}+4 c_{2}}, m_{+}=(|m+\mu|+$ $|m-\mu|) / 2$ and $P_{n}^{(a, b)}$ is the Jacobi polynomial. The $z$ component of the total angular momentum $m$, the total angular momentum $j$ take the quantized values as:

$$
\begin{aligned}
m & =-j,-j+1, \ldots, j-1, j \\
j & =\frac{|m+\mu|+|m-\mu|}{2}, \frac{|m+\mu|+|m-\mu|}{2}+1, \ldots
\end{aligned}
$$

The quantum numbers $j$ and $m$ explicitly depend on the Dirac quantization condition $\mu$. Note that depending on whether $\mu$ is half integral or integral, $j$ and $m$ respectively become half integer or integer. 
The radial differential equation is given by

$$
\frac{1}{r^{2}} \frac{d}{d r}\left(r^{2} \frac{d R}{d r}\right)-\frac{1}{r^{2}}\left(j+\frac{\delta_{1}+\delta_{2}}{2}\right)\left(j+\frac{\delta_{1}+\delta_{2}}{2}+1\right) R+2\left(E+\frac{1}{r}\right) R=0,
$$

which is exactly same as the radial equation for the hydrogen atom except that the orbital quantum number $l$ is replaced here by $j+\left(\delta_{1}+\delta_{2}\right) / 2$. The solution of Eq. (3.9) apart from normalization takes the form

$$
R_{n j}^{(\mu)}(r)=(2 \varepsilon r)^{j+\frac{\delta_{1}+\delta_{2}}{2}} e^{-\varepsilon r} F\left(-n+j+1 ; 2 j+\delta_{1}+\delta_{2}+2 ; 2 \varepsilon r\right),
$$

where $n=|\mu|+1,|\mu|+2, \ldots$ and the parameter $\varepsilon$ is given by

$$
\varepsilon=\sqrt{-2 E}=\frac{1}{n+\frac{\delta_{1}+\delta_{2}}{2}} .
$$

The exact form of the eigenvalue $E$ is now given by

$$
E \equiv E_{n}^{(\mu)}=-\frac{1}{2\left(n+\frac{\delta_{1}+\delta_{2}}{2}\right)^{2}} .
$$

\section{Supersymmetric generalized MIC-Kepler System}

We now come to the discussion of SUSY extension of generalized MIC-Kepler [16, 17] system, which is our interest in this work. Due to the close similarity of the radial equation (3.9) with the nonrelativistic Hydrogen atom radial equation, we will proceed with the same line as described in [8, 9, 10, 11, 12, for Hydrogen atom problem.

We first remove the first order derivative term from Eq. (3.9) by the transformation $R(r) \rightarrow \chi(r) / r$. The resulting differential equation suitable for the study of SUSY is

$$
-\frac{1}{2} \frac{d^{2} \chi(r)}{d r^{2}}+\left[-\frac{1}{r}-E+\frac{\left(j+\frac{\delta_{1}+\delta_{2}}{2}\right)\left(j+\frac{\delta_{1}+\delta_{2}}{2}+1\right)}{2 r^{2}}\right] \chi(r)=0 .
$$

Now we can construct bosonic and fermionic one dimensional Hamiltonian corresponding to the radial equation (4.1). We keep $j+\frac{\delta_{1}+\delta_{2}}{2}$ fixed for our calculation of SUSY. The partner Hamiltonian $H_{+}(r)$ for the generalized MIC-Kepler system may be read from Eq. (4.1) as

$$
H_{+}(r)=-\frac{1}{2} \frac{d^{2}}{d r^{2}}+V_{+}(r),
$$

where the SUSY partner potential $V_{+}(r)$ takes the form

$$
V_{+}(r)=-\frac{1}{r}+\frac{\left(j+\frac{\delta_{1}+\delta_{2}}{2}\right)\left(j+\frac{\delta_{1}+\delta_{2}}{2}+1\right)}{2 r^{2}}+\frac{1}{2\left(j+\frac{\delta_{1}+\delta_{2}}{2}+1\right)^{2}} .
$$

The potential $V_{+}(r)$ is constructed in such a way that the ground state eigenvalue of the Hamiltonian Eq. (4.2) becomes zero. Because, according to the condition of SUSY in Eq, (2.14), we need the ground state eigenvalue of the partner Hamiltonian $H_{+}$to be zero. The constant term $\frac{1}{2\left(j+\frac{\delta_{1}+\delta_{2}}{2}+1\right)^{2}}$ in Eq. (4.3) guarantees that the ground state eigenvalue of the Hamiltonian in Eq. (4.2) is zero for fixed angular momentum quantum number $j$. The expression for SUSY partner potential $V_{+}(r)$ in Eq. (4.3) exactly matches with the SUSY partner potential $V_{+}(r)$ of the Hydrogenic problem if $j+\frac{\delta_{1}+\delta_{2}}{2}$ in Eq. (4.3) is replaced by the orbital angular momentum quantum number $l[8,9,10,11,12$. The energy eigenvalue of the partner Hamiltonian $H_{+}(r)$ Eq. (4.2) takes the form

$$
E_{n}^{+}=\frac{1}{2\left(j+\frac{\delta_{1}+\delta_{2}}{2}+1\right)^{2}}-\frac{1}{2\left(n+\frac{\delta_{1}+\delta_{2}}{2}\right)^{2}} \quad \text { for, } n \geq j+1 .
$$


Note that the energy eigenvalue $E_{n}^{+}$of the partner Hamiltonian $H_{+}(r)$ does not reduce to the energy eigenvalue of the corresponding partner Hamiltonian of the Hydrogenic problem after replacement of $j+\frac{\delta_{1}+\delta_{2}}{2}$ by orbital angular momentum quantum number $l$ in Eq. (4.4) unlike SUSY partner potential $V_{+}(r)$. However for $\mu=c_{1}=c_{2}=0, E_{n}^{+}$of $\mathrm{Eq}$ (4.4) reduces to the corresponding eigenvalue of the Hydrogenic problem and the same is applicable for SUSY partner potential $V_{+}(r)$. From Eq. (2.3) and Eq. (4.3) we can work out the super-potential $W(r)$ as

$$
W(r)=\frac{1}{\sqrt{2}\left(j+\frac{\delta_{1}+\delta_{2}}{2}+1\right)}-\frac{j+\frac{\delta_{1}+\delta_{2}}{2}+1}{\sqrt{2} r} .
$$

One may note here also that the super-potential $W(r)$ exactly matches with the super-potential of the Hydrogenic problem [8, 9, 10, 11, 12] if $j+\frac{\delta_{1}+\delta_{2}}{2}$ in Eq. (4.5) is replaced by $l$. From Eq. (2.3) and Eq. (4.5) we can calculate the SUSY partner potential $V_{-}$as

$$
V_{-}(r)=-\frac{1}{r}+\frac{\left(j+\frac{\delta_{1}+\delta_{2}}{2}+1\right)\left(j+\frac{\delta_{1}+\delta_{2}}{2}+2\right)}{2 r^{2}}+\frac{1}{2\left(j+\frac{\delta_{1}+\delta_{2}}{2}+1\right)^{2}} .
$$

It is now obvious from the above discussion of super-potential $W(r)$ that we may get partner potential of the Hydrogenic problem from Eq. (4.6) by the same replacement. Similarly we may get the eigenvalue $E_{n}^{-}$of the SUSY partner Hamiltonian $H_{-}(r)$ from the relation Eq. (2.14) and Eq. (4.4). From the above analysis one can make the following important observations in addition to the non-relativistic coulomb problem discussed in $[8,9,10,11,12$.

\subsection{Observation 1}

Consider the situation $c_{1}=c_{2}=\mu=0$. In this case the Hamiltonian in Eq. (3.1) reduces to the Hydrogen atom problem. Putting the above condition in Eq. (4.3) and Eq. (4.6) we can immediately get the SUSY partner potentials $V_{\text {hydrogen+ }}(r)$ and $V_{\text {hydrogen- }}(r)$ respectively for the Hydrogen atom problem as

$$
\begin{aligned}
& V_{\text {hydrogen }+}(r)=-\frac{1}{r}+\frac{l(l+1)}{2 r^{2}}+\frac{1}{2(l+1)^{2}}, \\
& V_{\text {hydrogen- }}(r)=-\frac{1}{r}+\frac{(l+1)(l+2)}{2 r^{2}}+\frac{1}{2(l+1)^{2}},
\end{aligned}
$$

and we may also get the eigenvalues $\left(E_{\text {hydrogen }}\right)_{n}^{+}$and $\left(E_{\text {hydrogen }}\right)_{n}^{-}$for the Hydrogen atom problem, putting the same condition in Eq. (4.4) as

$$
\begin{aligned}
& \left(E_{\text {hydrogen }}\right)_{n}^{+}=\frac{1}{2(l+1)^{2}}-\frac{1}{2(n)^{2}} \quad \text { for, } \quad n \geq l+1 \\
& \left(E_{\text {hydrogen }}\right)_{n}^{-}=\left(E_{\text {hydrogen }}\right)_{n+1}^{+} \quad \text { for, } \quad n \geq l+1 .
\end{aligned}
$$

However the results Eq. (4.7) - Eq. (4.10) are not new to us. It has already been calculated in Ref. [8, 9, 10, 11, 12. Although the physical meaning of these results was explained in [8, 9, 10, 11, 12; ;ere we briefly discuss about that. Consider the case $l=0$ ( $s$ orbitals). We can see from Eq. (4.9) and Eq. (4.10) that the spectrum of $H_{-}($for $l=1)$ is same as that of $H_{+}($for $l=0)$ with the ground state removed. So $H_{-}$( for $l=1$ ) may describe a system with all the $s$ orbitals removed. It can have the following physical description. For lithium atom we may have this kind of situation where two of the three electrons of the lithium atom are in the $1 s$ orbital. So for lithium atom if the electron-electron interaction is taken to be zero and if the valence electron is far away moved from the core electrons then the effective interaction will look like Coulombic. Thus the $l=0$ levels of the lithium atom becomes the SUSY partner of the Hydrogen atom $l=0$ levels. 


\subsection{Observation 2}

Consider the situation $c_{1}=c_{2}=0$ of the Hamiltonian in Eq. (3.1). This is the two particle problem which have magnetic charges $g_{1}, g_{2}$ in addition to their electric charges $e_{1}, e_{2}$. The potential $V_{ \pm}(r)$ take the form

$$
\begin{aligned}
& V_{+}(r)=-\frac{1}{r}+\frac{j(j+1)}{2 r^{2}}+\frac{1}{2(j+1)^{2}}, \\
& V_{-}(r)=-\frac{1}{r}+\frac{(j+1)(j+2)}{2 r^{2}}+\frac{1}{2(j+1)^{2}},
\end{aligned}
$$

and the corresponding eigenvalues are given by

$$
\begin{aligned}
& E_{n}^{+}=\frac{1}{2(j+1)^{2}}-\frac{1}{2(n)^{2}} \text { for, } n \geq j+1 \\
& E_{n}^{-}=E_{n+1}^{+} \quad \text { for, } \quad n \geq j+1 .
\end{aligned}
$$

It is to be noted that the expressions in Eq. (4.11) - Eq. (4.14) look like Hydrogenic problem in Eq. (4.7) - Eq. (4.10), but it is not the same problem because $j$ can take values $j=|\mu|,|\mu|+1,|\mu|+2, \ldots$. , whereas for Hydrogenic problem $l$ can take non negative integral values only. If one considers the case of lowest value of $j$, i.e., $j=|\mu|$, then it is easy to show that the systems with Dirac quantization condition differing by $\left|\mu_{1}\right|-\left|\mu_{1}\right|=1$ are super-symmetric partners. Since $\mu$ can take values like $\mu=0, \pm \frac{1}{2}, \pm 1, \pm \frac{3}{2}, \ldots$, it is straightforward to conclude that system with $\mu= \pm \frac{1}{2}, \pm \frac{3}{2}, \ldots$ belong to one SUSY family and system with $\mu=0, \pm 1, \pm 2, \ldots$ belong to other SUSY family. The two family gets decoupled in this scenario. In the next subsection we will show that it is necessary to incorporate the additional potentials with the MIC-Kepler system, as it has been done in Refs. [16, 17, to get a unified SUSY family.

\subsection{Observation 3}

Finally, consider the situation when all the three parameters $\mu, c_{1}, c_{2}$ are nonzeros. The potential $V_{+}(r)$ and the respective energy eigenvalue $E_{n}^{+}$is given by Eq. (4.3) and Eq. (4.4) respectively. Now consider the situation when $\delta_{1}+\delta_{2}=1$. This can be achieved by appropriately tuning the constant parameter $c_{1}$ and $c_{2}$ in the Hamiltonian (3.1). Replacing $\delta_{1}+\delta_{2}=1$ in Eq. (4.3) and Eq. (4.4), we can see that systems with half integral Dirac quantization condition, $\mu= \pm \frac{1}{2}, \pm \frac{3}{2}, \ldots$. belong to the family of integral Dirac quantization condition.

\section{Conclusion}

SUSY has been shown to be a good symmetry for nonrelativistic coulomb system [8, 9, 10, 11, 12. It has been successfully applied to many other systems also, for example, in harmonic oscillator system [18, it has been applied to get supersymmetric system. We however show in our calculation that if SUSY is conserved in MIC-Kepler system, then there exist two kind of super-symmetric families, one with half integral Dirac quantization condition $\mu= \pm \frac{1}{2}, \pm \frac{3}{2}, \ldots$ and other with integral Dirac quantization condition $\mu=0, \pm 1, \pm 2, \ldots$. We also show that in order to unify these two kind of SUSY families into one, we need to generalize MIC-Kepler system [16, 17. Once we generalize, the two extra potentials with coefficients $c_{1}$ and $c_{2}$ in the Hamiltonian (3.1) allows us to unify the apparently separated SUSY families. We reproduce the non-relativistic coulomb result [8, 9, 10, 11, 12 in our formulation in subsection (4.1). The physics behind this SUSY can be understood from the SUSY problem of nonrelativistic Coulomb problem discussed in subsection (4.1). Since nonrelativistic Coulomb problem is a special case of Generalized MICKepler system, one can expect similar physics for Generalized MIC-Kepler system also. But in order to see this symmetry in nature, we first need dyon to exist in nature. SUSY breaking and self-adjointness is also an interesting issue [23. We hope to discuss this for generalized MIC-Kepler system in future. 


\section{Acknowledgments}

We thank Palash B. Pal for comments on manuscript and helpful discussions.

\section{References}

[1] P. Ramond, Phys. Rev. D 32415 (1971).

[2] Y. A. Golfand and E. P. Likhtman, JETP Lett. 13323 (1971).

[3] A. Neven and J. H. Schwarz, Nucl. Phys. B 3186 (1971).

[4] D. V. Volkov and V. P. Akulov, Phys. Lett. B 46109 (1973).

[5] J. Wess and B. Zumino, Nucl. Phys. B 7039 (1974).

[6] E. witten, Nucl. Phys. B 188513 (1981).

[7] F. Cooper, A. Khare and A. Sukhatme, Phys. Rept. 251267 (1995).

[8] V. A. Kostelecký and M. M. Nieto, Phys. Rev. Lett. 532285 (1984).

[9] V. A. Kostelecký and M. M. Nieto, Phys. Rev.. A 321293 (1985).

[10] V. A. Kostelecky, M. M. Nieto and D. R. Truax, Phys. Rev. D 322627 (1985).

[11] P. D. Jarvis and G. E. Stedman, J. Phys. A 191373 (1986).

[12] A. Bohm, Phys. Rev. D 333358 (1986).

[13] H. Katsura and H. Aloki, J. Math. Phys. 47032301 (2006).

[14] D. Zwanziger, Phys. Rev. 1761480 (1968).

[15] H. McIntosh and A. Cisneros, J. Math. Phys. 11896 (1970).

[16] L. Mardoyan, J. Math. Phys. 444981 (2003).

[17] L. Mardoyan, quant-ph/0310143 v1 (2003).

[18] F. Cooper, A. Khare and U. Sukhatme, Supersymmetry in Quantum Mechanics (World Scientific Publishing Company, 2002).

[19] F. Cooper and B. Freedman, Annals Phys. 146262 (1983).

[20] M. De Crombrugghe and V. Rittenberg, Annals Phys. 15199 (1983).

[21] B. K. Bagchi, Supersymmetry In Quantum and Classical Mechanics (Chapman and Hall, 2000).

[22] P. R. Giri, Mod. Phys. Lett. A22, 2365 (2007).

[23] H. Falomir, P. A. G. Pisani, J. Phys. A 384665 (2005). 